\title{
Desarrollo y Validación de una Escala de Actitudes hacia el Consumo y Legalización de la Marihuana en Población Mexicana
}

\author{
Development and Validation of a Scale of Attitudes towards the Consumption and \\ Legalization of Marijuana in the Mexican Population
}

\author{
Oscar Ulises Reynoso González ${ }^{1}$ Juan Francisco Caldera Montes ${ }^{2}$, Ignacio Pérez Pulido ${ }^{3}$, \\ María del Rosario Zamora Betancourt ${ }^{4}$, Jaime Fuentes-Balderrama ${ }^{5}$ y Samuel Alejandro Portillo Peñuelas ${ }^{6}$
}

\begin{abstract}
Resumen
El presente trabajo de investigación tuvo como propósito analizar la validez y confiabilidad de la Escala de Actitudes hacia el Consumo y Legalización de la Marihuana (EACLM). El estudio se desarrolló con una muestra incidental de 452 mexicanos de entre 18 y 45 años. Después de abordar la validez de contenido mediante jueces expertos, se ejecutó un análisis factorial exploratorio (máxima verosimilitud) y confirmatorio (máxima verosimilitud con errores robustos) para asegurar la validez de constructo de la escala; además, se calculó la consistencia interna mediante el coeficiente alfa de Cronbach. La versión final del instrumento quedó conformada por 16 reactivos agrupados en tres factores oblicuos: Consumo y legalización medicinal, Consumo recreativo y Legalización recreativa. Se recomienda el uso de la escala para conocer la actitud de distintos grupos sociales sobre el tema y sumar a la discusión nacional sobre el uso medicinal y recreativo del cannabis en México.
\end{abstract}

Palavras-chave: actitudes, consumo, legalización, marihuana, medición

\begin{abstract}
The purpose of this research was to analyze the validity and reliability of the Scale of Attitudes towards the Consumption and Legalization of Marijuana (EACLM). The study was carried out with an incidental sample of 452 Mexicans aged between 18 and 45 years old. After analysis of the content validity by expert judges, exploratory (maximum likelihood) and confirmatory factor analyses (maximum likelihood with robust errors) were conducted to ensure the scale's construct validity; in addition, internal consistency was calculated using Cronbach's alpha coefficient. The final version of the instrument contains 16 items grouped into three oblique factors: Medical use and legalization, Recreational use and Recreational legalization. The use of the scale is recommended to explore the attitudes of different social groups on the subject and add to national discussion on the medicinal and recreational use of cannabis in Mexico.
\end{abstract}

Keywords: attitudes, consumption, legalization, marijuana, measurement

\footnotetext{
${ }^{1}$ Doctor en Psicología, Maestro en Ciencias Sociales y Licenciado en Psicología. Profesor de asignatura en el Centro Universitario de Los Altos de la Universidad de Guadalajara. Av. Rafael Casillas Aceves No. 1200 Cp. 4762. Tel. 3787828033 ext. 56919. Tepatitlán de Morelos, Jalisco, México. Correo: ulises.reynoso@academicos.udg.mx. Orcid: http://orcid.org/0000-0002-0598-4665

${ }^{2}$ Doctor en Enseñanza de las Ciencias, Maestro en Educación y Licenciado en Psicología. Profesor de tiempo completo en el Centro Universitario de Los Altos de la Universidad de Guadalajara. Av. Rafael Casillas Aceves No. 1200 Cp. 47620. Tepatitlán de Morelos, Jalisco, México. Correo: jfcaldera@cualtos.udg.mx. Orcid: http://orcid.org/0000-0002-8999-3736

${ }^{3}$ Doctor en Educación, Maestro en Enseñanza de las Ciencias y Licenciado en Estudios Políticos y Gobierno. Profesor de tiempo completo en el Centro Universitario de Los Altos de la Universidad de Guadalajara. Av. Rafael Casillas Aceves No. 1200 Cp. 47620. Tepatitlán de Morelos, Jalisco, México. Correo: iperez@ cualtos.udg.mx. Orcid: https://orcid.org/0000-0002-8215-8850

${ }^{4}$ Doctora en Educación, Maestra en Educación y Licenciada en Psicología. Profesor de tiempo completo en el Centro Universitario de Los Altos de la Universidad de Guadalajara. Av. Rafael Casillas Aceves No. 1200 Cp. 47620. Tepatitlán de Morelos, Jalisco, México. Correo: rzamora@ cualtos.udg.mx. Orcid: http://orcid.org/0000-0002-2731-4208

${ }^{5}$ Doctor en Investigación Psicológica, Maestro en Orientación Psicológica y Licenciado en Psicología. Investigador asociado de la Escuela de Trabajo Social Steve Hicks de la Universidad de Texas en Austin. 1925 San Jacinto Blvd., Stop D3500, Austin, Texas, 78712. Correo: j.fuentes@austin.utexas.edu. ORCID: http://orcid.org/0000-0002-8225-0294

${ }^{6}$ Doctor en Educación, Maestro en Educación con Campo en Formación Docente y Licenciado en Educación Primaria. Profesor de asignatura en el Instituto Tecnológico de Sonora. 5 de febrero 818 Sur, Centro. CP.85000. Ciudad Obregón, Sonora, México. Correo:samuel.portillo40519@potros.itson.edu.mx. ORCID:http://orcid.org/0000-0002-1521-6619

Revista Iberoamericana de Diagnóstico y Evaluación - e Avaliação Psicológica. RIDEP · No61 · Vol.4 · 147-163 · 2021

ISSN: 1135-3848 print /2183-6051online
} 


\section{Introducción}

Según la Oficina de Las Naciones Unidas Contra la Droga y el Delito (UNODC) la marihuana es la droga más consumida en el mundo y la cantidad de consumidores sigue aumentando considerablemente (UNODC, 2018; 2021). En México, a pesar de su ilegalidad, el consumo de marihuana (al menos una vez) ha aumentado significativamente en población general. A saber, según la última Encuesta Nacional de Consumo de Drogas, Alcohol y Tabaco en México (2016-2017) se pasó de un $6.0 \%$ en 2011 a un $8.6 \%$ en 2016 de consumidores de marihuana, siendo considerablemente mayor en hombres (14.0\%) que en mujeres (3.7\%) (Instituto Nacional de Psiquiatría Ramón de la Fuente Muñiz et al., 2017). Respecto al año en curso, el Observatorio Mexicano de Salud Mental y Consumo de Sustancias Adictivas (2021) reporta una tendencia de aumento hacia el consumo de la marihuana a un $14.6 \%$, manteniéndose una mayor prevalencia de consumo de hombres sobre las mujeres.

Desde hace algunos años algunos países han cambiado su política sobre el consumo de marihuana y han legalizado su uso medicinal o recreativo. Ejemplo de ello son Uruguay, Países Bajos y Canadá (Excelsior, 2018). En Estados Unidos de América, aunque la ley federal prohíbe su consumo, se ha dotado de libertad a los Estados para regularizar su uso dentro de sus territorios. En México se ha debatido fuertemente sobre su legalización, lo que ha conllevado a distintos amparos para su uso recreativo o medicinal, incluso se ha sugerido legalizar la producción, venta y consumo en destinos turísticos (Valadez, 2018).

Respecto al debate sobre su legalización, los argumentos de tipo medicinal-terapéutico, jurídico, económico y de consumo como derecho (recreativo) son los que mayormente precisan las posiciones a favor o en contra de la legalización del cannabis (Hernández \& Lora, 2015). A saber, las posturas a favor, respecto a la cuestión de la frecuencia de consumo, sostienen que la marihuana es una droga inocua, inofensiva y relativamente no perjudicial contra la salud (incluso menos nociva que el tabaco y el alcohol, bajo el enfoque del margen de exposición), aumentando su consumo en medida en que es menor la percepción del riesgo, principalmente en muestras adolescentes (Lachenmeier \& Rehm, 2015; Moral, Rodríguez, \& Sirvent, 2006). Por su parte, quienes se enfocan en los efectos psicológicos, sostienen que son leves y no generan dependencia, por lo que su legalización podría incrementar la recaudación estatal y la reducción del narcotráfico, añadiendo las ventajas medicinales que posee sobre enfermedades crónico-degenerativas (Madoz \& Ochoa, 2014).

Finalmente, respecto a su legalización, algunos autores mantienen la idea del control de comercialización, calidad y producción de la marihuana, así como la posibilidad de incrementar el conocimiento epidemiológico y científico para mejorar la prevención de adicciones y la investigación de usos médicos mediante ensayos clínicos (Hernández \& Sotelo, 2013) siendo esta última postura la que se encuentra fuera de conflicto a debate y es la que mayormente hace empuje a su aprobación, pasando a la libertad de consumo en el aspecto legal y de nuevas formas de capitalizar la gran oferta y demanda en el plano económico (Hernández \& Lora, 2015).

Por otro lado, quienes mantienen una postura de rechazo, argumentan que la marihuana es la apertura a drogas más fuertes, cuestión que se evidencia en la iniciación y orden de consumo (de menor a mayor: marihuana, anfetamina, cocaína y heroína) siendo los jóvenes quienes realizan el consumo principalmente de manera grupal (Vega \& Aramendi, 2006). Respecto a sus efectos neuropsiquiátricos, Nizama (2017) afirma que el consumo de marihuana, aparte de la adicción, causa neurodegeneración, alteración del circuito endocannabinoide, sistema dopaminérgico, serotoninérgico y desregulación del circuito mesolímbico cortical.

A lo anterior, se añaden los episodios de psicosis y dependencia cuando su consumo se realiza durante el embarazo (Propospero, Ruiz, Cortés, Herrera, \& Méndez, 2019). Asimismo, de lograrse la legalización, Hernández y Sotelo (2013) proyectan que además de los daños a la salud, provocará el aumento en el consumo (principalmente en jóvenes), así como daños a terceros por conducir bajo el influjo de la marihuana con las subsecuentes alteraciones en habilidades motoras. Todo ello, ante la incertidumbre de saber si habrá un descenso de las 
actividades del narcotráfico (Moreno, 2015) y el impacto de la reglamentación del uso del cannabis con fines recreativos en los países y estados que han permitido su uso (UNODC, 2018). En este sentido, la postura jurídica de tensión entre el individuo y la colectividad es la que se encuentra mayormente arraigada en las posturas de rechazo al libre consumo de la marihuana (Hernández \& Lora, 2015).

Estos argumentos de tipo económico, político y médico, tanto a favor como en contra del consumo recreativo y medicinal de la marihuana, no solo juegan un papel importante en el tema de la legalización, sino también en el posicionamiento de los ciudadanos frente al debate. Este posicionamiento es sumamente relevante debido al peso que tiene la opinión ciudadana sobre la legalización (Serrano, 2015), especialmente en temas que pueden ser controversiales. Ante un escenario en el que se discuten aspectos favorables y desfavorables, tanto de la legalización como del consumo de cannabis, será pertinente poner atención a la postura de la comunidad. En dicho sentido, habría que identificar en un primer momento la opinión de los ciudadanos frente a la legalización, diferenciando el uso medicinal del uso recreativo $\mathrm{y}$, en un segundo momento, el posicionamiento sobre la posibilidad de consumir, tanto por cuestiones medicinales como recreativas.

Una forma adecuada de aproximarse a dicha trama es mediante la postura teórica de las actitudes, las cuales se definen como valoraciones que las personas construyen sobre distintos aspectos de la realidad, al analizar la información que reciben del exterior en torno a dimensiones evaluativas (Garrido \& Álvaro, 2007). Las actitudes desempeñan un conjunto de funciones imprescindibles a la hora de buscar, procesar y responder a la información sobre el entorno, ya que sirven como organizadores del conocimiento, dirigen el comportamiento, consolidan la identidad y la expresión de valores (Briñol, Falces, \& Becerra, 2007). En ocasiones las actitudes pueden ser ambivalentes, es decir, se puede evaluar un objeto tanto de forma positiva como negativa (Baron \& Byrne, 2005). Sobre las dimensiones de dicho constructo, múltiples autores concuerdan en señalar que las actitudes se componen de tres elementos, a saber, cognitivo (creencias), afectivo (los sentimientos) y conductual (la predisposición a actuar) (Fabrigar, MacDonald, \& Wegener, 2005; Insko \& Schopler, 1980). Éstas pueden adquirirse de otras personas a través del aprendizaje social, ya sea por condicionamiento clásico o instrumental o mediante el aprendizaje observacional (Baron \& Byrne, 2005).

Una de las ventajas de esta postura teórica es la posibilidad de desarrollar instrumentos para medir el grado de acuerdo o desacuerdo, de rechazo o aceptación sobre algún aspecto en específico, mejor conocidas como escalas de actitudes (Morales et al., 2003). Éstas permiten identificar el posicionamiento de los respondientes a través de un conjunto de reactivos o ítems que aluden a un objeto determinado.

En este caso, a partir de la discusión en torno a la marihuana, el objeto de actitud gira en torno a dos aspectos, en primer lugar, el aspecto del consumo, refiriendo aquellas creencias, sentimientos y disposiciones que giran en torno a la posibilidad de probar o consumir la marihuana, es decir, oportunidad, curiosidad o rechazo por ello. El segundo eje se encuentra centrado en la legalización y las creencias en torno a ésta, haciendo una delimitación entre la legalización completa que incluye el uso recreativo y, la legalización moderada con fines estrictamente medicinales.

En la literatura especializada sobre el tema en idioma español, existen una serie de instrumentos que pretenden medir la actitud hacia la marihuana, los cuales se enfocan principalmente en la intención conductual de consumo (García del Castillo et al., 2012; Lloret, Morell, Laguía, \& Moriano, 2018; Olivar \& Carrero, 2007). Estos instrumentos identifican a la diversión, relajación, reducción del malestar, creatividad, escalada, así como los problemas académicos, de salud y familiares como los principales factores que orillan al consumo en adolescentes y jóvenes (Olivar \& Carrero, 2007). Además de ello se abordan los distintos factores de riesgo y protección ante la permisividad o prohibición de los padres y el consumo o no consumo de los amigos. Ello, referente a tomar una actitud ante el nivel de exposición con personas que tienen apertura o rechazo al consumo de marihuana (García del Castillo et al., 2012). En algunos 
instrumentos que integran la predisposición para el uso y consumo, la percepción de riesgo y la satisfacción por el consumo como dimensiones a evaluar, se suele integrar en la misma categoría que la marihuana a otras sustancias (cocaína, metanfetaminas, estimulantes, etc.). En el caso de Lloret et al. (2018) integran en su escala la actitud hacia el consumo de cannabis (creencias en torno a su valoración y consumo), la norma subjetiva (influencia que el entorno inmediato a la persona ejerce sobre su conducta), la autoeficacia para la abstinencia (creencias sobre la medida en que la persona se siente capaz de no consumir cannabis en distintas situaciones) y la intención de consumo (la declaración del respondiente acerca de la posibilidad de uso).

Asimismo, se han identificado instrumentos en inglés que abordan el consumo problemático (Adamson \& Sellman, 2003; Bashford, Flett, \& Copeland, 2010; Legleye, Karila, Beck, \& Reynaud, 2007; Martin, Copeland, Gilmour, Gates, \& Swift, 2006), las expectativas y motivaciones por el consumo de cannabis (Lee, Neighbors, Hendershot, \& Grossbard, 2009; Schafer \& Brown, 1991) o la opinión de especialistas para su recomendación médica (Chan, Knoepke, Cole, McKinnon, \& Matlock, 2017). No obstante, ninguno de dichos instrumentos considera la dimensión de la legalización.

Del mismo modo, existen algunas aproximaciones al tema desde otras perspectivas, principalmente desde la teoría de las representaciones sociales (Bulla, Vasquez, \& Güichá, 2010; Dany \& Apostolidis, 2002; De Araújo, Castanha, Barros, \& Castanha, 2006; García et al., 2010; Henao, 2012). Bulla et al. (2010), por ejemplo, abordaron las representaciones sociales de un grupo de estudiantes universitarios colombianos, donde utilizaron como categorías de análisis la información general sobre la marihuana y su consumo, así como las actitudes y las experiencias. Con respecto a la categoría de actitudes, los participantes señalaron que la razón más fuerte por la cual las personas se abstienen de consumir está relacionada con el cúmulo de ideas negativas que a nivel social existe respecto de la marihuana y quienes la consumen, ya que ésta es vista de forma negativa. Del mismo modo, se cree que la gente no consume por ignorancia respecto a la marihuana, porque todavía no saben que en realidad no es tan negativa (como en el pasado se suponía), aunque también puede darse el caso de personas que no lo hacen simplemente porque no les interesa. En el mismo sentido, García et al. (2010) concluyeron que la representación social de la marihuana se construye a partir de las actitudes, prejuicios, creencias y la propia experiencia frente al consumo como tal.

Con respecto al tema de la legalización, la Cámara de Diputados en México, a través del Centro de Estudios Sociales y de Opinión Pública (CESOP, 2018), realizó una encuesta nacional sobre la legalización y uso de la marihuana y la amapola. Los resultados indicaron que el $86.8 \%$ de las 900 personas encuestadas manifestaron estar a favor de la legalización con fines medicinales, el $27.2 \%$ en pro del uso recreativo y $47.0 \%$ sobre el uso industrial. Al preguntarles sobre las consecuencias de su aprobación, el $64.9 \%$ espera que se incremente el número de personas adictas, $71.8 \%$ supone un mayor número de consumidores, $66.9 \%$ cree que habrá un incremento en la demanda de servicios de salud y el 51.8\% que aumentará la violencia en el país. Hablando de consumo medicinal individual, el $76.4 \%$ de las personas estaría de acuerdo en consumir marihuana ya que más del $80 \%$ de la muestra entrevistada considera que el cannabis tiene propiedades curativas y el $49 \%$ confía en que cura enfermedades. Por último, solo el $26.0 \%$ de los respondientes considera que México se encuentra preparado para regular el uso de la marihuana.

Los referentes empíricos citados con anterioridad confirman el interés por abordar la actitud, representación, postura u opinión de distintos grupos sociales sobre la marihuana. No obstante, se observa que los instrumentos centran su análisis en la predisposición o intención conductual de consumo, la permisividad o prohibición de los padres, el consumo de personas cercanas, las expectativas y motivaciones por el consumo, la percepción de riesgo y la satisfacción por el consumo, pero sin integrar el tema de la legalización y sin separar los distintos tipos de consumo. Además, como se mencionó con anterioridad, en algunos casos en los que se integra la actitud o la predisposición hacia el 
consumo, se incluye en la medición otro tipo de sustancias. Esto no permite conocer la actitud específica hacia el consumo de marihuana y tampoco diferenciar entre el uso medicinal o recreativo. En lo que respecta al tema de la legalización, es más frecuente el uso de encuestas (pregunta única) o cuestionarios y no de escalas, lo cual puede representar un problema para identificar correctamente la actitud del respondiente. Como señalan Morales et al. (2003), utilizar un conjunto de reactivos, en lugar de una pregunta directa sobre el tema, brinda una mejor medición sobre constructos complejos, dota de mayor validez a las respuestas, aumenta la fiabilidad de medida y permite identificar de mejor manera las diferencias entre las posturas de los individuos.

En tal sentido, la presente investigación se propuso como objetivo principal desarrollar una escala de actitudes hacia el consumo y legalización de la marihuana que considere el uso recreativo y para fines medicinales. Del mismo modo se evaluaron sus propiedades psicométricas, tanto de validez y confiabilidad mediante un análisis factorial exploratorio y confirmatorio.

\section{Método}

\section{Participantes}

Para conformar la muestra del estudio fue publicado un formulario con el instrumento en diversas plataformas y redes sociales. Se utilizó en un primer momento un espacio de interacción virtual entre estudiantes del Servicio Nacional del Bachillerato en Línea (conocido como Sala/café) que tiene por objetivo la interacción entre estudiantes y la vinculación con sus redes sociales. En la publicación, se solicita la participación en el estudio y compartir el enlace con sus familiares y amigos. Se eligió tal espacio debido a que es consultado por miles de estudiantes de diversas edades de todos los rincones del país $\mathrm{y}$, al solicitar que el formulario fuera compartido, se integraban participantes que no formaban parte de la plataforma. Fue necesario realizar una depuración de las respuestas obtenidas. Particularmente, se eliminaron aquellas que presentaron inconsistencias o errores de llenado $\mathrm{y}$ se mantuvieron únicamente las respuestas de los participantes de entre 18 y 45 años debido a que, al trabajar con variables cognitivas como las actitudes, puede haber diferencias en función de la edad (y no se contó con suficientes participantes para un análisis de invarianza). En dicho sentido, se constituyó una muestra incidental o por conveniencia (Hernández-Sampieri, Fernández-Collado \& Baptista-Lucio, 2014) de 452 mexicanos, provenientes de 28 de los 32 estados de la República Mexicana (60.2\% mujeres y 39.8\% hombres), con edades entre los 18 y 45 años $(M E=27, D E=7.1)$. La Tabla 1 muestra las características principales de la muestra.

Tabla 1.Características de la muestra

\begin{tabular}{lcc}
\hline & Frecuencia & Porcentaje \\
\hline Sexo & & \\
Mujeres & 272 & $60.2 \%$ \\
Hombres & 180 & $39.8 \%$ \\
Escolaridad* & & \\
$\quad$ Primaria & 5 & $1.1 \%$ \\
Secundaria & 310 & $68.6 \%$ \\
Preparatoria & 124 & $27.4 \%$ \\
Licenciatura & 13 & $2.9 \%$ \\
Estado civil & & \\
Soltero & 288 & $63.7 \%$ \\
Casado & 164 & $36.3 \%$ \\
Tiene hijos & & \\
Sí & 238 & $52.7 \%$ \\
No & 214 & $47.3 \%$ \\
*Grado terminado & &
\end{tabular}

\section{Instrumentos}

Escala de Actitudes hacia el Consumo y Legalización de la Marihuana (EACLM)

El instrumento quedó conformado en su versión final por 16 reactivos y tres factores oblicuos: Consumo y legalización Medicinal, Consumo recreativo y Legalización recreativa. El formato de respuesta utilizado fue de escalamiento ordinal (Likert) con cinco opciones de respuesta: (1) Completamente en desacuerdo; (2) En desacuerdo; (3) Ni de acuerdo ni desacuerdo; (4) De acuerdo; y (5) Completamente de acuerdo. Todos los reactivos cuentan con una puntuación directa, por lo que al incrementarse los valores se infiere una actitud más positiva a la legalización referida a las circunstancias expuestas en cada factor.

\section{Cuestionario dicotómico}

Adicionalmente se le preguntó a cada uno de los respondientes, en un formato dicotómico, su 
postura ante la legalización y consumo de la marihuana, tanto medicinal como recreativa a través de cuatro preguntas: (1) ¿Estás de acuerdo con la legalización de la marihuana con fines medicinales?; (2) ¿Estás de acuerdo con la legalización de la marihuana con fines recreativos?; (3) En caso de ser legal ¿Consumirías marihuana con fines medicinales?; (4) En caso de ser legal ¿Consumirías marihuana con fines recreativos?

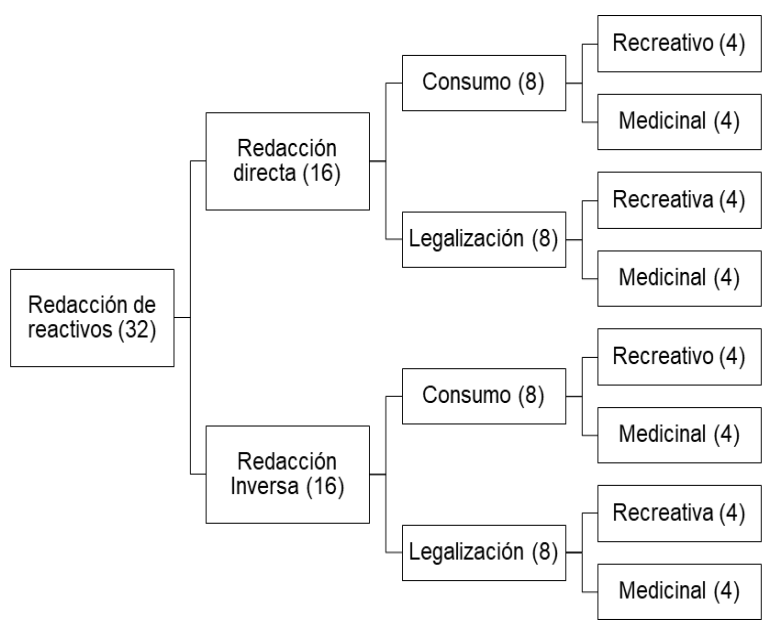

Figura 1. Distribución del contenido de los reactivos (Fuente: elaboración propia)

\section{Procedimiento}

De acuerdo con Ato, López y Benavente (2013), el diseño de la investigación es de tipo instrumental, ya que atañe a la elaboración y revisión de las propiedades psicométricas de la Escala de Actitudes hacia la Legalización y Consumo de Marihuana. De manera agregada, respecto a lo sugerido por Clark y Watson (2019), en primera instancia se realizó la delimitación del constructo y la búsqueda de la literatura sobre el tema, en donde se encontraron algunos instrumentos que abordaban la actitud hacia la marihuana, pero que se centraban en el consumo y no en la legalización. Enseguida se realizó un banco de 32 reactivos que contenían distintos aspectos sobre la legalización y el consumo recreativo y medicinal de la marihuana, tanto en sentido directo como inverso. Para su redacción se procuró que cada uno de ellos aludiera a un elemento específico de la teoría tripartita de las actitudes, es decir, que su contenido implicará una creencia, predisposición o emoción sobre el objeto de actitud junto a la temática de consumo o legalización (tanto recreativa como medicinal), usando un lenguaje simple, con una sola idea por reactivo, evitando los coloquialismos y procurando su brevedad (Clark \& Watson, 2019). La Figura 1 muestra la distribución temática de los reactivos propuestos.

Un aspecto importante por determinar fue el formato de respuesta del instrumento. Se consideró en primera instancia el escalamiento de Thurstone, el cual consiste en valorar los reactivos a partir del juicio de expertos de acuerdo con el grado de intensidad que expresan sobre el objeto de actitud. También se consideró el formato de diferencial semántico, solicitando que el respondiente valorará a través de adjetivos opuestos su posicionamiento sobre el consumo y la legalización. Sin embargo, se optó por usar el escalamiento tipo Likert con cinco opciones de respuesta (dos en cada polaridad y una neutra), ya que dicho método permite estimar de forma sencilla el posicionamiento del individuo, su interpretación es clara, requiere de un menor esfuerzo en comparación a los otros métodos citados y permite la obtención de instrumentos con alta consistencia interna (Garrido \& Álvaro, 2007), sin olvidar que, al ser el formato de respuesta más utilizado, los respondientes se encuentran mayormente familiarizados a su aplicación (Morales, Urosa, \& Blanco, 2003).

Enseguida, se revisó la validez de contenido mediante la evaluación de cuatro jueces expertos. En tal proceso se calculó la V de Aiken y se modificaron distintos reactivos de acuerdo con la opinión de los evaluadores. De igual manera, se realizó un pilotaje con un grupo de participantes para subsanar posibles errores de redacción o comprensión del instrumento. Al observarse dificultades y confusiones para responder los reactivos inversos, se optó por suprimirlos del análisis, dejando una versión preliminar de 16 reactivos. La recolección de información se realizó a través de un formulario de Google entre los meses de agosto y diciembre de 2020.

\section{Consideraciones éticas}

Como parte inicial del formulario, se mostraba un consentimiento informado que enunciaba los objetivos del estudio. Además, se hacía mención de que la participación era voluntaria, que podían abandonar el cuestionario en cualquier momento (por cualquier motivo) y 
que se resguardará el anonimato y la confidencialidad de sus datos e información. Como medida adicional, se dejó el correo de contacto del equipo de investigación para que los respondientes pudieran solicitar información más detallada del estudio o de su involucramiento. Únicamente pudieron participar aquellos que aceptaban los términos y condiciones expuestos anteriormente.

\section{Análisis de datos}

Para propósitos del proceso de validación, y siguiendo las recomendaciones para validación cruzada propuestas por diversos autores (Anderson \& Gerbing, 1988; Brown, 2006; Lloret-Segura et al., 2014), se utilizó una submuestra para explorar una solución factorial que posteriormente sería confirmada con una segunda submuestra. Consecuentemente, la muestra fue segmentada aleatoriamente en dos partes para la ejecución del Análisis Factorial Exploratorio $(n=227)$ y el Análisis Factorial Confirmatorio $(\mathrm{n}=225)$. Se evaluó la normalidad uni y multivariada de los datos para determinar si había necesidad de manejar los datos extremos y evaluar si se cumplían los supuestos estadísticos para correr pruebas de hipótesis multivariadas sin inflar la tasa de error tipo I.

El análisis factorial exploratorio y de consistencia interna (Alpha de Cronbach) se desarrolló utilizando la primera submuestra. Dado que los ítems presentaban 5 categorías de respuesta, se cumplía el supuesto normalidad univariada aproximada y se buscaba probar relaciones lineales entre factores $\mathrm{e}$ ítems ordinales, se eligió factorizar la matriz de correlaciones policóricas (Lloret-Segura et al., 2014). Se determinó la adecuación muestral por medio de la prueba Kaiser Meyer Olkin, donde valores por encima de .8 son considerados como satisfactorios. La capacidad de la matriz de correlación para ser factorizable se determinó por medio de la prueba de esfericidad de Bartlett, donde un valor significativo descarta la presencia de una matriz de identidad. Los factores se extrajeron por medio del método Máxima Verosimilitud (MV), ya que se ha evidenciado que gracias a su robustez brinda resultados adecuados frente a la falta de normalidad multivariada cuando los ítems siguen una distribución univariada aproximadamente normal (LloretSegura et al., 2014; Onyekachi \& Olanrewaju, 2020; Oyeyemi \& Ipinyomi, 2010). Se determinó el número de factores siguiendo el criterio de Kaiser (i.e. eigenvalues mayores a uno) y confirmando con un análisis paralelo de Horn. Dado que se esperaban correlaciones moderadas/altas entre factores, se asumió la oblicuidad entre dimensiones y la solución factorial fue sometida a la rotación promax.

El análisis factorial confirmatorio se llevó a cabo con la segunda submuestra y dada la ausencia de normalidad multivariada, el modelo fue calculado utilizando el estimador Máxima Verosimilitud con errores estándar Robustos ("MLR", por sus siglas en inglés). El ajuste absoluto del modelo se determinó utilizando la chi cuadrada ajustada (Satorra \& Bentler, 2001), donde valores no significativos indican concordancia absoluta entre las matriz de covarianza esperada y la observada. El ajuste cercano se midió utilizando los índices de ajuste cercano (CFI) y Tucker Lewis (TLI), donde valores superiores a .95 indican un excelente ajuste. Los residuales se cuantificaron con el error cuadrático medio de aproximación (RMSEA) y el residual promedio estandarizado (SRMR), donde valores menores a .08 reflejan un ajuste cercano (Kline, 2015). De manera complementaria se estimaron los índices de fiabilidad compuesta (IFC) y la varianza media extraída (VME) para cada uno de los factores, donde valores IFC $>.7$ y VME $>.5$ son deseables. Con la finalidad de probar el modelo obtenido por medio del análisis factorial exploratorio, se contrastaron los índices de ajuste para este modelo contra una estructura de un factor y otro a dos factores.

Se evaluó la sensibilidad y la especificidad de la escala a través de las preguntas del cuestionario dicotómico “¿Estás de acuerdo con la legalización de la marihuana con fines medicinales?”, “¿Estás de acuerdo con la legalización de la marihuana con fines recreativos?" y "En caso de ser legal, ¿consumirías marihuana con fines recreativos?", utilizando el gráfico AUC (Area Under the Curve o Área bajo la curva). Tal gráfico evalúa el grado en que las puntuaciones de la escala identifican adecuadamente las actitudes del respondiente a partir de un juicio externo (en este caso las preguntas dicotómicas). Valores cercanos a 1 indicarán que el 
Tabla 2. Ítems originales, valores de $\mathrm{V}$ de Aiken y modificaciones efectuadas

\begin{tabular}{|c|c|c|}
\hline Reactivo original & V de Aiken & Reactivo modificado \\
\hline $\begin{array}{l}\text { 1. Legalizar la marihuana para uso medicinal es una opción } \\
\text { adecuada. }\end{array}$ & .94 & $\begin{array}{l}\text { 1. Legalizar la marihuana para uso medicinal en mi país es } \\
\text { una buena opción. }\end{array}$ \\
\hline $\begin{array}{l}\text { 2. Legalizar el consumo recreativo de la marihuana es algo } \\
\text { positivo. }\end{array}$ & 1.00 & $\begin{array}{l}\text { 2. Es positivo que se legalice el consumo recreativo de la } \\
\text { marihuana. }\end{array}$ \\
\hline $\begin{array}{l}\text { 3. Legalizar la marihuana como remedio terapéutico es } \\
\text { aceptable. }\end{array}$ & .94 & $\begin{array}{l}\text { 3. Legalizar la marihuana como remedio terapéutico para } \\
\text { algunas enfermedades es aceptable. }\end{array}$ \\
\hline $\begin{array}{l}\text { 4. Me gustaría que el consumo recreativo de la marihuana } \\
\text { fuera algo permitido legalmente. }\end{array}$ & 1.00 & $\begin{array}{l}\text { 4. Me gustaría que el consumo recreativo de la marihuana } \\
\text { fuera permitido legalmente. }\end{array}$ \\
\hline $\begin{array}{l}\text { 5. Aprobar el consumo de marihuana con fines medicinales } \\
\text { traerá beneficios a la salud. }\end{array}$ & .88 & $\begin{array}{l}\text { 5. Aprobar el consumo de marihuana con fines medicinales } \\
\text { mejorará el tratamiento de algunas enfermedades. }\end{array}$ \\
\hline $\begin{array}{l}\text { 6. Creo que la legalización de la marihuana con fines } \\
\text { recreativos sería un éxito. }\end{array}$ & .69 & $\begin{array}{l}\text { 6. Creo que la legalización de la marihuana con fines } \\
\text { recreativos sería un avance como sociedad. }\end{array}$ \\
\hline $\begin{array}{l}\text { 7. Apoyaría una propuesta de ley sobre el uso de marihuana } \\
\text { con fines medicinales. }\end{array}$ & 1.00 & $\begin{array}{l}\text { 7. Apoyaría una propuesta de ley sobre el uso de marihuana } \\
\text { con fines medicinales. }\end{array}$ \\
\hline $\begin{array}{l}\text { 8. La sociedad está lista para la legalización del uso } \\
\text { recreativo de la marihuana. }\end{array}$ & .94 & $\begin{array}{l}\text { 8. La sociedad está lista para la legalización del uso } \\
\text { recreativo de la marihuana. }\end{array}$ \\
\hline $\begin{array}{l}\text { 9. Estoy dispuesto a consumir marihuana con fines } \\
\text { recreativos. }\end{array}$ & 1.00 & 9. Consumiría marihuana con fines recreativos. \\
\hline $\begin{array}{l}\text { 10. Si favorece a mi salud, consumiría marihuana como } \\
\text { remedio medicinal. }\end{array}$ & 1.00 & $\begin{array}{l}\text { 10. } \mathrm{Si} \text { favorece a mi salud, consumiría marihuana como } \\
\text { tratamiento médico. }\end{array}$ \\
\hline $\begin{array}{l}\text { 11. Siento curiosidad por consumir marihuana de forma } \\
\text { recreativa. }\end{array}$ & .94 & $\begin{array}{l}\text { 11. Siento curiosidad por consumir marihuana de forma } \\
\text { recreativa. }\end{array}$ \\
\hline $\begin{array}{l}\text { 12. Consumiría marihuana con fines medicinales si } \\
\text { padeciera de dolores fuertes. }\end{array}$ & 1.00 & $\begin{array}{l}\text { 12. Consumiría marihuana con fines medicinales si } \\
\text { padeciera de dolores fuertes. }\end{array}$ \\
\hline $\begin{array}{l}\text { 13. Si se diera la oportunidad consumaría marihuana por } \\
\text { diversión. }\end{array}$ & 1.00 & $\begin{array}{l}\text { 13. Si se diera la oportunidad consumaría marihuana por } \\
\text { diversión. }\end{array}$ \\
\hline $\begin{array}{l}\text { 14. Si el doctor lo recomienda, consumiría marihuana con } \\
\text { fines medicinales sin dudarlo. }\end{array}$ & 1.00 & $\begin{array}{l}\text { 14. Si el doctor lo recomienda, consumiría marihuana como } \\
\text { medicamento. }\end{array}$ \\
\hline $\begin{array}{l}\text { 15. Consumir marihuana de forma recreativa puede ser algo } \\
\text { agradable. }\end{array}$ & .94 & $\begin{array}{l}\text { 15. Consumir marihuana de forma recreativa puede ser } \\
\text { agradable. }\end{array}$ \\
\hline $\begin{array}{l}\text { 16. Creo que no tendría problema en consumir marihuana } \\
\text { con fines medicinales a favor de mi salud. }\end{array}$ & 1.00 & $\begin{array}{l}\text { 16. Creo que no tendría problema en consumir marihuana } \\
\text { con fines medicinales. }\end{array}$ \\
\hline
\end{tabular}

instrumento es capaz de detectar correctamente tanto las actitudes favorables como desfavorables, evitando los falsos positivos o negativos (Cerda \& Cifuentes, 2012; Donis, 2012). Por último, se abordó el poder discriminativo de los ítems a través de la comparación de los grupos con mayor y menor puntuación, así como contrastando las medias entre de cada reactivo y factor con las respuestas del cuestionario dicotómico. Los análisis factoriales exploratorios y confirmatorios se llevaron a cabo en R v.4.0.1 utilizando los paquetes Lavaan, psych y semPlot. La detección de puntos de apalancamiento y el área bajo la curva de clasificación se hicieron en SPSS v.25, la normalidad multivariada se calculó usando el paquete MVR de R v.4.0.1.

\section{Resultados}

\section{Validez de contenido}

Como sustento de validez de contenido de la EACLM se realizó un proceso de evaluación por jueces para todos los reactivos. Se eligieron tanto jueces con experiencia en procesos de validación de instrumentos como a investigadores que trabajaban el tema de la legalización o el consumo de sustancias. Fue solicitado que para cada ítem se emitiera una calificación sobre la claridad, pertinencia y representatividad para el constructo que pretendían medir. A partir de estas evaluaciones se calculó la llamada V de Aiken (Aiken, 1980; Escurra, 1988), la cual expresa la concordancia entre los expertos. En cada uno de los reactivos se encontraron puntuaciones satisfactorias $(\mathrm{V}>.80)$, únicamente un reactivo presentó niveles inferiores, por lo cual fue modificado a través del consenso de los jueces. En la Tabla 2 se muestran los reactivos originales, los valores de $\mathrm{V}$ de Aiken obtenidos y las modificaciones realizadas a partir de los comentarios de los evaluadores.

\section{Análisis Factorial Exploratorio y consistencia interna}

Para la submuestra exploratoria, los ítems presentaron una distribución univariada aproximadamente normal (Asimetría< $<2 \mid$ y curtosis $<|6|)$ (Curran, West, \& Finch, 1996). Sin embargo, los coeficientes de Mardia demostraron ausencia de normalidad multivariada $\left(M_{\text {Kurt }}=28.51, p<.00 ; \quad M_{\text {skew }}=2367.02, p<.00\right)$. Se 
Tabla 3. Subescalas, cargas factoriales y consistencias internas.

\begin{tabular}{|c|c|c|c|c|}
\hline $\begin{aligned} & \text { Subescala/Ítem } \\
& \text { Consumo y legalización medicinal }(\alpha=.94)\end{aligned}$ & $\mathrm{F} 1$ & $\mathrm{~F} 2$ & F3 & $h^{2}$ \\
\hline 10. Si favorece a mi salud, consumiría marihuana como tratamiento médico & 0.94 & -0.18 & 0.17 & 0.85 \\
\hline 12. Consumiría marihuana con fines medicinales si padeciera de dolores fuertes & 0.94 & -0.16 & 0.17 & 0.87 \\
\hline 14. Si el doctor lo recomienda, consumiría marihuana como medicamento & 0.91 & -0.06 & 0.13 & 0.89 \\
\hline 16. Creo que no tendría problema en consumir marihuana con fines medicinales & 0.85 & -0.10 & 0.06 & 0.67 \\
\hline 1. Legalizar la marihuana para uso medicinal en mi país es una buena opción & 0.80 & 0.15 & -0.08 & 0.75 \\
\hline 3. Legalizar la marihuana como remedio terapéutico para algunas enfermedades es aceptable & 0.78 & 0.16 & -0.14 & 0.69 \\
\hline 7. Apoyaría una propuesta de ley sobre el uso de marihuana con fines medicinales & 0.76 & 0.21 & -0.18 & 0.66 \\
\hline $\begin{array}{l}\text { 5. Aprobar el consumo de marihuana con fines medicinales mejorará el tratamiento de } \\
\text { algunas enfermedades }\end{array}$ & 0.75 & 0.19 & -0.06 & 0.72 \\
\hline \multicolumn{5}{|l|}{ Consumo recreativo $(\alpha=.91)$} \\
\hline 13. Si se diera la oportunidad consumaría marihuana por diversión & 0.02 & 0.95 & -0.03 & 0.87 \\
\hline 9. Consumiría marihuana con fines recreativos & -0.06 & 0.88 & 0.04 & 0.77 \\
\hline 11. Siento curiosidad por consumir marihuana de forma recreativa & -0.01 & 0.78 & 0.15 & 0.78 \\
\hline 15. Consumir marihuana de forma recreativa puede ser agradable & 0.10 & 0.69 & 0.15 & 0.74 \\
\hline \multicolumn{5}{|l|}{ Legalización recreativa $(\alpha=.91)$} \\
\hline 8. La sociedad está lista para la legalización del uso recreativo de la marihuana & 0.07 & 0.09 & 0.86 & 0.95 \\
\hline 2. Es positivo que se legalice el consumo recreativo de la marihuana & 0.05 & 0.07 & $\mathbf{0 . 8 3}$ & 0.82 \\
\hline $\begin{array}{l}\text { 6. Creo que la legalización de la marihuana con fines recreativos sería un avance como } \\
\text { sociedad }\end{array}$ & 0.03 & 0.14 & 0.76 & 0.78 \\
\hline 4. Me gustaría que el consumo recreativo de la marihuana fuera permitido legalmente & -0.03 & 0.27 & 0.60 & 0.62 \\
\hline
\end{tabular}

Nota. Muestra AFE (n=227); Método de extracción=Máxima Verosimilitud con rotación oblicua (Promax); Matriz patrón

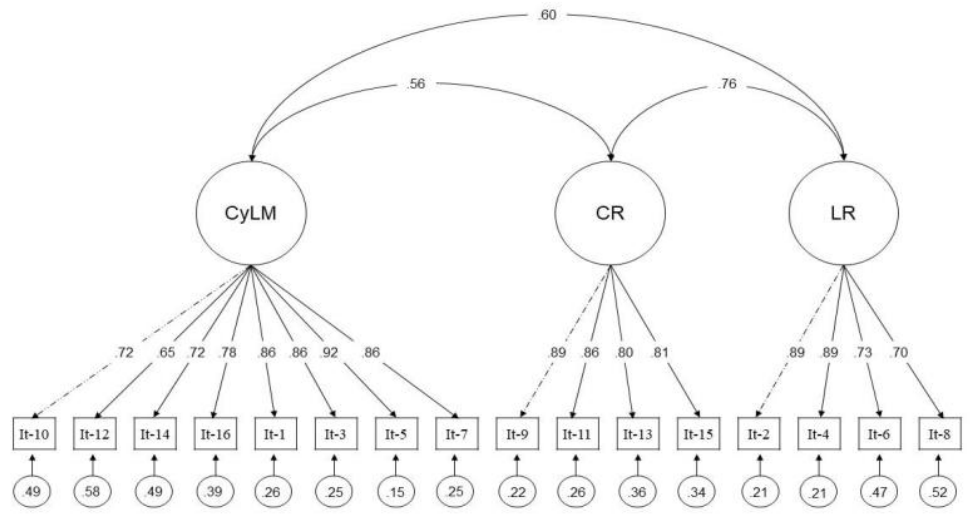

Figura 2. Análisis Factorial Confirmatorio de la EALCM

Nota. $\mathrm{CyLM}=$ Consumo y legalización medicinal; $\mathrm{CR}=$ Consumo recreativo $\mathrm{LR}=$ Legalización recreativa.

Fuente: Elaboración propia a partir de semPlot

tomó la decisión de correr los análisis sin transformar los datos, pues la distancia de Cook descartó la necesidad de manejar o suprimir los casos extremos, ya que éstos no actuaban como puntos de apalancamiento $\left(\mathrm{D}_{\mathrm{i}} \mathrm{Cook}>.02\right)$. El análisis arrojó un KMO de .93, indicativo de adecuación muestral, así como una esfericidad significativa que es indicativa de una matriz factorizable (Abad, Olea, Ponsoda, \& García, 2011). Las comunalidades observadas excedieron .55; la estimación convergió satisfactoriamente tras 4 iteraciones $\mathrm{y}$, bajo el criterio de Kaiser, arrojó una solución factorial de 3 dimensiones con autovalores mayores a 1 que explican el $78 \%$ de la varianza $(37 \%, 21 \%$ y $19 \%$ respectivamente). El arreglo de mediciones, cargas factoriales, comunalidades y consistencias internas se pueden observar en la Tabla 3.
La primera dimensión agrupó a los reactivos de consumo y de legalización con fines estrictamente medicinales $(14,12,7,10,5,1,16$ y 3). En cambio, la segunda y tercera dimensión integraron los reactivos sobre el uso recreativo de la marihuana, primero sobre el aspecto de consumo $(9,11,13$ y 15) y segundo sobre la legalización $(6,4,2$ y 8). Las correlaciones entre factores se encuentran entre .52 y $\quad .68 \quad \mathrm{La}$ consistencia interna total de la EACLM es de .94 y la consistencia de cada factor es superior a .91, por lo cual se le cataloga como un instrumento con alta consistencia interna, así como con validez de constructo (ver Tabla 3). 
Tabla 4. Pesos factoriales, Varianza explicada y Fiabilidad compuesta

\begin{tabular}{|c|c|c|c|c|}
\hline Subescala/Ítem & $\lambda$ & $V E$ & $V M E$ & $I F C$ \\
\hline Consumo y legalización medicinal & & & .64 & .93 \\
\hline Ítem 10 & .72 & .52 & & \\
\hline Ítem 12 & .65 & .42 & & \\
\hline Ítem 14 & .72 & .52 & & \\
\hline Ítem 16 & .78 & .61 & & \\
\hline Ítem 1 & .86 & .74 & & \\
\hline Ítem 3 & .86 & .74 & & \\
\hline Ítem 5 & .92 & .85 & & \\
\hline Ítem 7 & .86 & .74 & & \\
\hline Consumo recreativo & & & .71 & .91 \\
\hline Ítem 9 & .89 & .79 & & \\
\hline Ítem 11 & .86 & .74 & & \\
\hline Ítem 13 & .80 & .64 & & \\
\hline Ítem 15 & .81 & .66 & & \\
\hline Legalización recreativa & & & .65 & .88 \\
\hline ítem 2 & .89 & .79 & & \\
\hline ítem 4 & .89 & .79 & & \\
\hline ítem 6 & .73 & .53 & & \\
\hline Ítem 8 & .70 & .49 & & \\
\hline
\end{tabular}

Tabla 5. Actitudes hacia la marihuana

\begin{tabular}{|c|c|c|c|c|}
\hline Factores de la EACLM & $M$ & $D E$ & Sí & No \\
\hline Consumo y legalización medicinal & 3.93 & .92 & & \\
\hline Consumo recreativo & 2.46 & 1.14 & & \\
\hline Legalización recreativa & 3.03 & 1.11 & & \\
\hline \multicolumn{5}{|l|}{ Preguntas del cuestionario } \\
\hline ¿Estás de acuerdo con la legalización de la marihuana con fines medicinales? & & & $385(85.2 \%)$ & $67(14.8 \%)$ \\
\hline En caso de ser legal ¿Consumirías marihuana con fines medicinales? & & & $343(75.9 \%)$ & $109(24.1 \%)$ \\
\hline En caso de ser legal ¿Consumirías marihuana con fines recreativos? & & & $117(25.9 \%)$ & $335(74.1 \%)$ \\
\hline ¿Estás de acuerdo con la legalización de la marihuana con fines recreativos? & & & $192(42.5 \%)$ & $260(57.5 \%)$ \\
\hline
\end{tabular}

\section{Análisis Factorial Confirmatorio}

Para identificar si la solución factorial es una representación fiel de los constructos en otra muestra, se llevaron a cabo tres análisis factoriales confirmatorios con la segunda submuestra a fin de contrastar el ajuste contra un modelo a uno y dos factores respectivamente. De manera similar, los coeficientes de Mardia demostraron ausencia de normalidad multivariada $\left(M_{K u r t}=35.30, \quad p<.00\right.$; $\left.M_{\text {skew }}=2604.07, p<.00\right)$. Ya que la distancia de Cook nuevamente descarta que los datos extremos fungen como punto de apalancamiento $\left(D_{i}\right.$ Cook>.02). En tal sentido, se tomó la decisión de correr los análisis sin transformar los datos, pero optar por un estimador robusto a la no-normalidad multivariada. El modelo se puede ver en la Figura 2.

El modelo de la solución factorial obtenida por medio del análisis factorial exploratorio obtuvo S-B $\chi^{2}$ (101): $189.68(p<.000)$, CFI: .96, TLI: .95, RMSEA: .07** [IC .06-.09], SRMR: .04 , AIC:8937.06, BIC:9056.78, indicando un ajuste cercano bueno, así como niveles aceptables de discrepancia entre la matriz de covarianza observada y la teórica. Todos los factores presentaron varianzas latentes significativas, así como cargas factoriales altas y significativas. Las varianzas explicadas para cada ítem fueron altas $\left(\mathrm{R}^{2}>.42\right)$ y se obtuvieron valores satisfactorios de Varianza media extraída e índices de fiabilidad compuesta (Tabla 4).

En contraste, el modelo alternativo a un factor obtuvo S-B $\chi^{2}$ (104): 757.1 ( $\left.p<.000\right)$, CFI: .67, TLI: .62, RMSEA: .17** [IC .16-.18], SRMR: .14, AIC:9691.99, BIC:9801.45. El modelo a dos factores se estimó englobando el consumo y legalización medicinal en un factor, así como el consumo y legalización recreativa en el segundo. Este segundo modelo alternativo a dos factores obtuvo S-B $\chi^{2}$ (103): 300 ( $\left.p<.000\right)$, CFI: .89, TLI: .88, RMSEA: .09** [IC .08-.10], SRMR: .06, AIC:9100.05, BIC:9212.93. Ambos modelos alternativos presentan un ajuste significativamente menor (i.e. $\Delta \chi^{2}(\Delta \mathrm{gl})>\chi^{2}$ crit.) y por ende se descartan. Estos resultados ofrecen evidencia a favor de la solución factoriade tres dimensiones 
Tabla 6. Comparación entre factores y reactivos con las preguntas dicotómicas

\begin{tabular}{|c|c|c|c|c|c|c|c|}
\hline & \multicolumn{2}{|c|}{ Sí } & \multicolumn{2}{|c|}{ No } & \multirow{2}{*}{$t$} & \multirow{2}{*}{$g l$} & \multirow{2}{*}{ Sig. } \\
\hline & $M$ & $D E$ & $M$ & $D E$ & & & \\
\hline Consumo y legalización medicinal & 4.20 & .64 & 2.36 & .69 & 21.21 & 450 & .000 \\
\hline Ítem 1 & 4.15 & .91 & 2.28 & .98 & 15.16 & 450 & .000 \\
\hline Ítem 3 & 4.30 & .89 & 2.99 & 1.02 & 10.92 & 450 & .000 \\
\hline Ítem 5 & 4.29 & .79 & 2.90 & .90 & 12.90 & 450 & .000 \\
\hline Ítem 7 & 4.25 & .75 & 2.28 & 1.02 & 18.51 & 450 & .000 \\
\hline
\end{tabular}

2. En caso de ser legal ¿Consumirías marihuana con fines medicinales?

\begin{tabular}{|c|c|c|c|c|c|c|c|}
\hline & \multicolumn{2}{|c|}{ Sí } & \multicolumn{2}{|c|}{ No } & \multirow{2}{*}{$t$} & \multirow{2}{*}{$g l$} & \multirow{2}{*}{ Sig. } \\
\hline & $M$ & $D E$ & $M$ & $D E$ & & & \\
\hline Consumo y legalización medicinal & 4.29 & .58 & 2.78 & .85 & 20.95 & 450 & .000 \\
\hline Ítem 10 & 4.27 & .81 & 2.53 & 1.14 & 17.61 & 450 & .000 \\
\hline Ítem 12 & 4.39 & .78 & 2.64 & 1.20 & 17.69 & 450 & .000 \\
\hline Ítem 14 & 4.26 & .74 & 2.42 & 1.10 & 19.79 & 450 & .000 \\
\hline Ítem 16 & 4.17 & .84 & 2.44 & 1.13 & 17.22 & 450 & .000 \\
\hline
\end{tabular}

3. En caso de ser legal ¿Consumirías marihuana con fines recreativos?

\begin{tabular}{|c|c|c|c|c|c|c|c|}
\hline & \multicolumn{2}{|c|}{ Sí } & \multicolumn{2}{|c|}{ No } & \multirow[b]{2}{*}{$t$} & \multirow[b]{2}{*}{$g l$} & \multirow{2}{*}{ Sig. } \\
\hline & $M$ & $D E$ & $M$ & $D E$ & & & \\
\hline Consumo recreativo & 3.83 & .78 & 1.98 & .80 & 21.57 & 450 & .000 \\
\hline Ítem 9 & 3.97 & .87 & 1.90 & .99 & 19.99 & 450 & .000 \\
\hline Ítem 11 & 3.79 & 1.03 & 1.93 & 1.02 & 16.99 & 450 & .000 \\
\hline Ítem 13 & 3.58 & 1.16 & 1.79 & .94 & 16.63 & 450 & .000 \\
\hline Ítem 15 & 3.98 & .841 & 2.30 & 1.05 & 15.58 & 450 & .000 \\
\hline
\end{tabular}

4. ¿Estás de acuerdo con la legalización de la marihuana con fines recreativos?

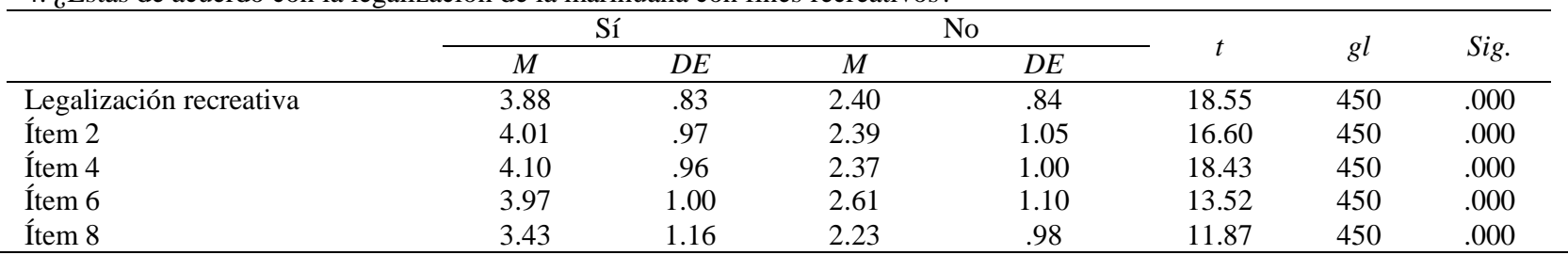

Nota. el factor Marihuana medicinal es presentado en dos preguntas debido a que integró los reactivos sobre consumo y legalización con fines terapéuticos.

obtenida en el análisis factorial exploratorio, mostrando puntuaciones confiables y válidas en población mexicana.

\section{Poder discriminativo de los ítems}

Como última fase del proceso de validación, se constató el poder discriminativo de los ítems mediante el cálculo de los valores $t$ que resultan de comparar las medias obtenidas en cada uno de los ítems por los sujetos en los grupos "bajo" (27\% de sujetos con puntuaciones más bajas de cada factor) y "alto" ( $27 \%$ de sujetos con puntuaciones más altas en la misma condición). En todos los casos se presentaron diferencias estadísticamente significativas entre los grupos $(t(242)>12.24, p=.000)$. En la Tabla 5 se presentan los estadísticos descriptivos, tanto de los factores del instrumento como de las preguntas del cuestionario dicotómico. Las puntuaciones de los factores se obtuvieron al promediar los reactivos que los constituían, a fin de obtener valores que oscilaran entre 1 y 5 en cada dimensión. En todos los casos, valores cercanos a 5 serán sinónimo de actitudes positivas, mientas que los cercanos a 1 indicarán actitudes negativas o desfavorables.

Como medida adicional, se contrastaron las medias de cada dimensión entre las respuestas dicotomizadas de las participantes obtenidas mediante el cuestionario. En el primer caso, fueron generados dos grupos a partir de las respuestas a la pregunta ¿Estás de acuerdo con la legalización de la marihuana con fines medicinales?; enseguida se compararon los promedios de tales grupos en los reactivos que aludían a dicho contenido (1,3,5 y 7), así como su factor (Marihuana medicinal). Este proceso se repitió en cada una de las preguntas del cuestionario dicotómico. Como se muestra en la Tabla 6, se confirmaron diferencias significativas entre las respuestas positivas y negativas en los reactivos y factores de cada pregunta, confirmando una correcta discriminación de los ítems. 
Finalmente, se utilizaron las puntuaciones promedio en cada factor y su respectiva pregunta dicotómica para analizar su sensibilidad y especificidad a través del gráfico AUC. Para el caso del factor Consumo y legalización medicinal se obtuvo un área bajo la curva de .95 , indicando una excelente capacidad discriminativa. En Consumo recreativo el valor fue de .94 , también indicativo de una alta capacidad discriminativa y en Legalización recreativa de .89, que pese a ser la más baja de las tres, también demuestra una capacidad discriminativa adecuada.

\section{Discusión}

Aunque la redacción de los ítems contempló inicialmente la consolidación de cuatro factores, la actitud hacia el consumo y la legalización de la marihuana con fines medicinales fue agrupada en un solo factor, constituyendo entonces tres dimensiones. Independientemente de esta cuestión, el instrumento mostró propiedades psicométricas satisfactorias al agrupar congruentemente el contenido referente a la marihuana medicinal.

Por tanto, después de pasar por los procesos de validación de contenido (mediante jueces expertos), de constructo (a través de un análisis factorial exploratorio y confirmatorio) y de consistencia interna (alfa de Cronbach), es posible constatar que la EACLM es un instrumento válido y confiable para población mexicana y que permite identificar tres dimensiones sobre el tema en cuestión, a saber, Consumo y legalización medicinal (entendida como la postura hacia el consumo y la legalización del cannabis con fines terapéuticos), Consumo recreativo (que aborda la apertura personal al uso lúdico o libre de la marihuana) y Legalización recreativa (que atañe al posicionamiento del respondiente sobre la aprobación para su uso libre). Adicionalmente, el análisis discriminativo de los ítems y la AUC dotaron al instrumento de una adecuada validez de criterio.

$\mathrm{Si}$ bien identificar las actitudes hacia el consumo y la legalización hacia la marihuana no era parte del objetivo de investigación, pudo detectarse que las personas tienen en su mayoría una actitud positiva hacia la marihuana medicinal, ya que el $85.2 \%$ de los respondientes afirmó estar de acuerdo con su legalización y el $75.9 \%$ con el consumo. En suma, el factor de Legalización y consumo de marihuana obtuvo el promedio más alto $(M=3.93, \quad D E=.92)$. Dicha condición probablemente ocurra debido a que en buena parte de la población ya existe la experiencia de que la marihuana se emplea como "remedio medicinal" (como por ejemplo para mitigar dolores articulares), o en su caso, que ya ha permeado en ciertos sectores la perspectiva de quienes afirman que mediante el empleo de conocimientos especializados (científicos) se pueden controlar sus efectos nocivos (Propospero et al., 2019).

Una situación diferente se presentó en el ámbito recreacional. Por un lado, el factor de Legalización recreativa se mostró ligeramente más negativo, ya que el $42.5 \%$ de los participantes así lo manifestaron. Esta disminución también se vio reflejada en la media de dicho factor $(M=3.03$, $D E=1.11$ ), indicando que las personas rechazan en mayor medida la posibilidad de volver legal el consumo recreativo de marihuana. Por otro lado, la dimensión de Consumo recreativo se mostró sensiblemente más polarizada, dado que el $74.1 \%$ manifestó su desacuerdo a la posibilidad de consumir marihuana por diversión, curiosidad o pasatiempo. De hecho, fue este factor el que presentó el promedio más bajo de la escala $(M=2.46, D E=1.14)$. Dichos resultados pueden deberse a que en una considerable proporción de los mexicanos podría permear la idea de que con la legalización recreativa de la marihuana aumentará su consumo (principalmente en los más jóvenes) y, por ende, habrá más daños a la salud de la población, se propiciarán más accidentes (principalmente automovilísticos) y se incrementará el número de actos delincuenciales (Hernández \& Sotelo, 2013).

Las diferencias en las actitudes hacia el consumo y legalización de la marihuana recreativa pueden explicar el surgimiento de factores independientes para cada caso. Mientras que es probable que el surgimiento de un solo factor para la marihuana medicinal sea reflejo de una actitud más congruente por parte de los participantes hacia su uso y legalización. Tal condición reafirma el hecho de que las actitudes (en este caso hacia la marihuana) tienen su origen en el 
mundo social que envuelve a los individuos (Baron \& Byrne, 2005).

De hecho, estos resultados son bastante similares a los reportados por el CESOP (2018), ya que el $86.8 \%$ de las personas encuestadas en su estudio manifestaron estar a favor de la legalización con fines medicinales, mientras que solo el $27.2 \%$ apoyaba su regulación para uso recreativo. Incluso, refiriéndose al consumo medicinal, los hallazgos fueron similares, ya que el $76.4 \%$ de las personas estaría de acuerdo en consumir marihuana con fines medicinales.

A partir de la validación de la EACLM y los resultados encontrados, se sugiere que en investigaciones posteriores se analice la actitud hacia el consumo y la legalización de la marihuana de distintos grupos sociales, a fin de sumar la perspectiva de los mexicanos a la discusión nacional sobre el uso medicinal y recreativo del cannabis. Del mismo modo, sería pertinente el uso de la escala en otros países y contextos, sobre todo en aquellos que se encuentren disonantes con el tema en cuestión y requieren de conocer la actitud de sus ciudadanos al respecto.

A su vez, valdría la pena conocer la forma en que diversas variables se encuentran asociadas a estas actitudes, tanto de carácter sociodemográfico, como puede ser la edad, el sexo, el nivel de estudios o el nivel socioeconómico, así como con aspectos relacionados con el acercamiento al cannabis, es decir, si haber consumido previamente marihuana (ya sea de forma medicinal o recreativa) se asocia a una actitud más positiva o negativa hacia la legalización. Incluso, conocer si la frecuencia de consumo, la experiencia (positiva o negativa al consumir) o el acercamiento a personas consumidoras se encuentra vinculado a tener un mayor rechazo o aceptación hacia la marihuana. Además, al abordar su vínculo con el consumo, podría valorarse la posibilidad de crear programas preventivos para la drogodependencia (FontMayolas, Gras, \& Planes, 2013). Del mismo modo, podría abordarse su vínculo con otras variables psicosociales, como la actitud hacia la autoridad institucional (Gálvez, Salvo, Trizano, Hederich, Polanco, 2017) o el apoyo social.

La investigación también contó con algunas limitaciones, por lo cual los resultados deben ser interpretados con precaución. Dada la recopilación virtual de los datos y pese al anonimato en el proceso, las respuestas pueden estar sujetas al sesgo de auto reporte y factores de deseabilidad social. La variabilidad en la edad de los participantes también es un factor para considerar, pues la escala puede comportarse de manera distinta entre jóvenes y adultos; desafortunadamente, el tamaño de la muestra no permitió establecer la equivalencia psicométrica entre grupos de edad. Finalmente, si bien se presenta evidencia psicométrica de ajuste al modelo y consistencia interna, el método de extracción de factores por máxima verosimilitud es robusto mas no infalible en el uso de variables ordinales y datos que no sigan una distribución multivariada normal.

En conclusión, la EACLM es un instrumento de medición válido y confiable que sirve para cuantificar las actitudes de participantes mexicanos con respecto al consumo y legalización medicinal, el consumo recreativo y la legalización recreativa de la marihuana.

\section{Referencias}

Abad, F., Olea, J., Ponsoda, V., \& García, C. (2011). Medición en ciencias sociales y de salud. Madrid: Síntesis.

Adamson, S., \& Sellman, J. (2003). A prototype screening instrument for cannabis use disorder: The Cannabis Use Disorders Identification Test (CUDIT) in an alcoholdependent clinical sample. Drug Alcohol Rev, 22(3), 309-315.

https://doi.org/10.1080/0959523031000154454

Aiken, L. (1980). Content validity and reliability of single items or questionnaire. Educational and Psychological Measurement 40(4), 955959.

http://doi.org/10.1177\%2F001316448004000419

Anderson, J. C., \& Gerbing, D. W. (1988). Structural equation modeling in practice: A review and recommended two-step approach. Psychological Bulletin, 103(3), 411-423.

https://doi.org/10.1037/0033-2909.103.3.411

Ato, M., López, J., \& Benavente, A. (2013). Un sistema de clasificación de los diseños de 
investigación en psicología. Anales de psicología,29(3), 1038-1059.

http://dx.doi.org/10.6018/analesps.29.3.178511

Baron, R., \& Byrne, D. (2005). Psicología social. Madrid: Pearson.

Bashford, J., Flett, R., \& Copeland, J. (2010). The Cannabis Use Problems Identification Test (CUPIT): Development, reliability, concurrent and predictive validity among adolescents and adults. Addiction, 105(4), 615-625.

http://doi.org/10.1111/j.1360-

0443.2009.02859.x

Briñol, P., Falces, C., \& Becerra, A. (2007). Actitudes. En J. Morales, E. Gaviria, M. Moya, \& I. Cuadrado (Coord.), Psicología social (págs. 457-490). Madrid: McGraw-Hill.

Brown, T. A. (2006). Confirmatory factor analysis for applied research. The Guilford Press.

Bulla, A., Vasquez, A., Güichá, A., ManriqueAbril, F., \& Ospina, J. (2010). Representaciones sociales del consumo de marihuana en estudiantes universitarios. Psychologia. Avances de la disciplina, 4(1), 89-101. http://doi.org/10.21500/19002386.1161

Cerda, J., \& Cifuentes, L. (2012) Uso de curvas ROC en investigación clínica. Aspectos teórico-prácticos. Revista Chilena de Infectología 29(2).

http://dx.doi.org/10.4067/S0716-

10182012000200003

CESOP. (2018). Legalización y uso de la marihuana y la amapola 2018. México: Cámara de Diputados, Centro de Estudios Sociales y de Opinión Pública. Obtenido de https://bit.ly/2QvOAsf

Chan, M., Knoepke, C., Cole, M., McKinnon, J., \& Matlock, D. (2017). Colorado medical students' attitudes and beliefs about marijuana. Journal of General Internal Medicine, 32(4), 458-463. http://doi.org/10.1007/s11606-016-3957-y

Clark, L. A., \& Watson, D. (2019). Constructing validity: New developments in creating objective measuring instruments. Psychological Assessment, 31(12), 14121427. http://doi.org/10.1037/pas0000626

Curran, P., West, S., \& Finch, J. (1996). The robustness of test statistics to nonnormality and specification error in confirmatory factor analysis. Psychological Methods, 1(1), 16-29. http://psycnet.apa.org/doi/10.1037/1082989X.1.1.16

Dany, L., \& Apostolidis, T. (2002). L'étude des représentations sociales de la drogue et du cannabis: Un enjeu pour la prévention. Sante Publique, 14(4), 335-344.

https://doi.org/10.3917/spub.024.0335

De Araújo, L., Castanha, A., Barros, A., \& Castanha, C. (2006). Estudo das representações sociais da maconha entre agentes comunitários de saúde. Ciencia Colectiva, 11(3), 827-836

http://www.redalyc.org/pdf/630/63011330.pdf

Donis, J. (2012). Evaluación de la validez y confiabilidad de una prueba diagnóstica. Avances en Biomedicina, 1(2), 73-81. http://erevistas.saber.ula.ve/index.php/biomed icina/article/view/4066

Escurra, L. (1988). Cuantificación de la validez de contenido por criterio de jueces. Revista de Psicología, 6(1-2), 103-111.

http://revistas.pucp.edu.pe/index.php/psicolog ia/article/view/4555

Excelsior. (17 de Octubre de 2018). Checa los países donde es legal el consumo de mariguana. Excelsior (en línea). Obtenido de http://www.excelsior.com.mx/global/checalos-paises-donde-es-legal-el-consumo-demariguana/1272316

Fabrigar, L., MacDonald, T., \& Wegener, D. (2005). The structure of attitudes. En D. Albarracín, B. Johnson, \& M. Zanna (Eds.), The handbook of attitudes (págs. 79-124). New Jersey: Lawrence Erlbaum Associates, Inc.

Font-Mayolas, S., Gras, M., \& Planes, M. (2013). El proceso de valoración de programas preventivos en drogodependencias. Revista Iberoamericana de Diagnóstico y Evaluación - e Avaliação Psicológica, 1(35), 183-199. http://www.redalyc.org/articulo.oa?id=4596/4 59645435010

Gálvez, J., Salvo, S., Trizano, Í., Hederich, C., \& Polanco, K. (2017). Equivalencia factorial de la Escala de Actitudes hacia la Autoridad Institucional en Adolescentes (AAI-A) Chilenos y Colombianos. Revista Iberoamericana de Diagnóstico y Evaluación 
- e Avaliação Psicológica, 1(46), 109-119. http://doi.org/10.21865/RIDEP46.1.08

García del Castillo, J., Dias, P., Díaz, J., Bastos, A., García del Castillo, Á., López, C., \& Maciá, D. (2012). Adaptación de las escalas de actitudes hacia el tabaco, alcohol y otras drogas en adolescentes portugueses. Salud y Drogas, 12(1), 79-99.

http://www.redalyc.org/articulo.oa?id=839/83 924615005

García, A., Torres, D., Barrios, F., Palacio, V., Rubio, A., \& Ugarte, L. (2010). Representación social del consumo de marihuana en un grupo de jóvenes universitarios consumidores de la Universidad Tecnológica de Pereira. Revista Médica de Risaralda, 16(2), 25-30. http://doi.org/10.22517/25395203.811

Garrido, A., \& Álvaro, J. L. (2007). Psicología social: Perspectivas psicológicas $y$ sociológicas. Madrid: McGraw-Hill.

Henao, S. (2012). Representaciones sociales del consumo de drogas en un contexto universitario, Medellín, Colombia, 2000. Revista Facultad Nacional De Salud Pública, 30(1), 26-37.

http://revistas.udea.edu.co/index.php/fnsp/arti cle/view/10587

Hernández, A., \& Lora, J. (2015). Marihuana: el debate entre la legalización y las políticas prohibicionistas en México. En A. Hernández (Ed.), Delito y mercancía. Despenalización y poder. El debate sobre la marihuana en México (pp. 68-133). México: Benemérita Universidad de Puebla. Obtenido de https://bit.ly/3eUE9rr

Hernández, S., \& Sotelo, J. (2013). Argumentos para el debate sobre la legalización de la marihuana en México. Entreciencias: diálogos en la Sociedad del Conocimiento, 1(2), 93-100.

http://dx.doi.org/10.21933/J.EDSC.2013.02.0 45

Hernández-Sampieri, R., Fernández-Collado, C., \& Baptista-Lucio, P. (2014).Metodología de la investigación. México: McGraw Hill.

Insko, C., \& Schopler, J. (1980). Psicología social experimental. México: Trillas.

Instituto Nacional de Psiquiatría Ramón de la Fuente Muñiz, Instituto Nacional de Salud
Pública, Comisión Nacional Contra las Adicciones \& Secretaría de Salud. (2017). Encuesta Nacional de Consumo de Drogas, Alcohol y Tabaco 2016-2017: Reporte de drogas. México: INPRFM. https://bit.ly/3hBYtj9

Kline, R. B. (2015). Principles and practice of structural equation modeling. Guilford publications

Lachenmeier, D. W., \& Rehm, J. (2015). Comparative risk assessment of alcohol, tobacco, cannabis and other illicit drugs using the margin of exposure approach. Scientific Reports, 5(8126), 1-7.

https://doi.org/10.1038/srep08126

Lee, C., Neighbors, C., Hendershot, C., \& Grossbard, J. (2009). Development and preliminary validation of a Comprehensive Marijuana Motives Questionnaire. Journal of Studies on Alcohol and Drugs, 70(2) 279-287. http://doi.org/10.15288/jsad.2009.70.279

Legleye, S., Karila, L., Beck, F., \& Reynaud, M. (2007). Validation of the CAST, a general population Cannabis Abuse Screening Test. Journal of Substance Use, 12(4), 233-242. http://doi.org/10.1080/14659890701476532

Lloret-Segura, S., Ferreres-Traver, A., HernándezBaeza, A., \& Tomás-Marco, I. (2014). El análisis factorial exploratorio de los ítems: Una guía práctica, revisada y actualizada. Anales de Psicología, 30(3), 1151-1169. https://doi.org/10.6018/analesps.30.3.199361

Lloret, D., Morell, R., Laguía, A., \& Moriano, J. (2018). Diseño y validación de una escala de intención de consumo de cannabis (CUIQ) para adolescentes. Adicciones, 30(1), 54-65. http://doi.org/10.20882/adicciones.865

Madoz, A., \& Ochoa, E. (2014). Legalización del cannabis: Argumentos a favor y en contra. Revista de Patología Dual, 1(1), 1-7. http://patologiadual.es/docs/revista/pdfs/2014 _3.pdf

Martin, G., Copeland, J., Gilmour, S., Gates, P., \& Swift, W. (2006). The Adolescent Cannabis Problems Questionnaire (CPQ-A): Psychometric properties. Addictive Behaviors, 31(12), 2338-2348.

http://doi.org/10.1016/j.addbeh.2006.03.001

Moral, M., Rodríguez, F., \& Sirvent, C. (2006). Factores relacionados com las actitudes 
juveniles hacia el consumo de alcohol y otras sustancias psicoactivas. Psicothema, 18(1), 52-58.

http://www.psicothema.es/pdf/3175.pdf

Morales, P., Urosa, B., \& Blanco, Á. (2003). Construcción de escalas de actitudes tipo Likert. Madrid: La Muralla.

Moreno, C. (2015). Despenalización de las drogas en México. En A. Hernández (Ed.), Delito y mercancía. Despenalización y poder. El debate sobre la marihuana en México (pp. 134-173). México: Benemérita Universidad de Puebla. Obtenido de https://bit.ly/3eUE9rr

Nizama, M. (2017). Desmitificación del uso medicinal de la marihuana: Argumentos médicos, científicos y sociales en contra de su legalización. Acta Médica Peruana, 34(3), 231-236.

http://www.redalyc.org/articulo.oa?id=966533 89012

Observatorio Mexicano de Salud Mental y Consumo de Sustancias Psicoactivas (2021) Informe sobre la situación de la salud mental y el consumo de sustancia psicoactivas en México. Recuperado de https://www.gob.mx/salud\%7Cconadic/docu mentos/91969

Olivar, A., \& Carrero, V. (2007). Análisis de la intención conductual de consumir cannabis en adolescentes: Desarrollo de un instrumento de medida basado en la teoría de la conducta planificada. Trastornos Adictivos, 9(3), 161236.

http://doi.org/10.1016/S15750973\%2807\%2975645-3

Onyekachi, A., \& Olanrewaju, S. (2020) A comparison of principal component analysis, maximum likelihood and the principal axis in factor analysis. American Journal of Mathematics and Statistics 10(2), 44-54. http://doi.org/10.5923/j.ajms.20201002.03

Oyeyemi, G., \& Ipinyomi, R. (2010). Some robust methods of estimation in factor analysis in the presence of outliers. ICASTOR Journal of Mathematical Sciences 4(1), 1-12. $\mathrm{http} / / / \mathrm{www}$. icastorindia.org/view_details.aspx ?fn $=246$

Propospero, O., Ruiz, A., Cortés, J., Herrera, A., \& Méndez, M. (2019). Marihuana: Legalización y atención médica. Revista de la
Facultad de Medicina de la UNAM, 62(6), 623.

http://doi.org/10.22201/fm.24484865e.2019.6 2.6.02

Satorra, A., \& Bentler, P. M. (2001). A scaled difference chi-square test statistic for moment structure analysis. Psychometrika, 66(4), 507514.

Schafer, J., \& Brown, S. (1991). Marijuana and cocaine effect expectancies and drug use patterns. Journal of Consulting and Clinical Psychology, 59(4), 558-565.

http://doi.org/10.1037//0022-006x.59.4.558

Serrano, A. (2015). La participación ciudadana en México. Estudios Políticos 9(34), 93-116. http://www.scielo.org.mx/pdf/ep/n34/n34a5.p df

UNODC. (2018). Informe Mundial Sobre las Drogas 2018. Nueva York: Organización de las Naciones Unidas. Oficina de las Naciones Unidas contra la Droga y el Delito. Obtenido de

https://www.unodc.org/wdr2018/index.html

UNODC. (2021). Informe Mundial Sobre las Drogas 2021. Viena: Organización de las Naciones Unidas. Oficina de las Naciones Unidas contra la Droga y el Delito. Obtenido de https://www.unodc.org/unodc/en/data-andanalysis/wdr2021.html

Valadez, R. (25 de enero de 2018). Titular de SECTUR plantea legalizar mota en zonas turísticas. Milenio (en línea). Obtenido de http://www.milenio.com/negocios/titularsectur-plantea-legalizar-mota-zonas-turisticas

Vega, A., \& Aramendi, P. (2011). Las drogas en los Centros de Iniciación Profesional: Aportaciones de algunos estudios del País Vasco. Intervención psicoeducativa en la desadaptación social: IPSE-ds, 4(1), 5769.https://ipseds.ulpgc.es//PSEds_volumen_4_ART_4.pdf 


\section{Anexo I}

\section{Escala de Actitudes hacia el consumo y legalización de la marihuana (EALCM)}

A continuación, se presentan una serie de oraciones que pretenden conocer tu opinión acerca del uso y legalización del cannabis o marihuana, tanto con fines medicinales como recreativos. Los enunciados que mencionan el uso MEDICINAL DE LA MARIHUANA se refieren a su empleo como medicamento para tratar algunas enfermedades. Por otro lado, cuando se habla del uso RECREATIVO DE LA MARIHUANA implica su uso libre o por diversión (como sucede con el alcohol o el tabaco).

Lee atentamente cada una de ellas y coloca una X según tu grado de acuerdo o desacuerdo con cada una de las oraciones. Es importante mencionar que no existen respuestas correctas o incorrectas, lo importante es que respondas con toda honestidad. Tu participación será completamente anónima y la información que proporciones será utilizada únicamente con fines académicos.

\begin{tabular}{|c|c|c|c|c|c|}
\hline Ítems & $\begin{array}{l}\text { Completamente } \\
\text { en desacuerdo }\end{array}$ & En desacuerdo & $\begin{array}{l}\text { Ni de acuerdo ni } \\
\text { en desacuerdo }\end{array}$ & De acuerdo & $\begin{array}{c}\text { Completamente } \\
\text { de acuerdo }\end{array}$ \\
\hline \multicolumn{6}{|l|}{$\begin{array}{l}\text { 1. Legalizar la marihuana para uso } \\
\text { medicinal en mi país es una buena opción. }\end{array}$} \\
\hline \multicolumn{6}{|l|}{$\begin{array}{l}\text { 2. Es positivo que se legalice el consumo } \\
\text { recreativo de la marihuana. }\end{array}$} \\
\hline \multicolumn{6}{|l|}{$\begin{array}{l}\text { 3. Legalizar la marihuana como remedio } \\
\text { terapéutico para algunas enfermedades es } \\
\text { aceptable. }\end{array}$} \\
\hline \multicolumn{6}{|l|}{$\begin{array}{l}\text { 4. Me gustaría que el consumo recreativo } \\
\text { de la marihuana fuera permitido } \\
\text { legalmente. }\end{array}$} \\
\hline \multicolumn{6}{|l|}{$\begin{array}{l}\text { 5. Aprobar el consumo de marihuana con } \\
\text { fines medicinales mejorará el tratamiento } \\
\text { de algunas enfermedades. }\end{array}$} \\
\hline \multicolumn{6}{|l|}{$\begin{array}{l}\text { 6. Creo que la legalización de la marihuana } \\
\text { con fines recreativos sería un avance como } \\
\text { sociedad. }\end{array}$} \\
\hline \multicolumn{6}{|l|}{$\begin{array}{l}\text { 7. Apoyaría una propuesta de ley sobre el } \\
\text { uso de marihuana con fines medicinales. }\end{array}$} \\
\hline \multicolumn{6}{|l|}{$\begin{array}{l}\text { 8. La sociedad está lista para la } \\
\text { legalización del uso recreativo de la } \\
\text { marihuana. }\end{array}$} \\
\hline \multicolumn{6}{|l|}{$\begin{array}{l}\text { 9. Consumiría marihuana con fines } \\
\text { recreativos. }\end{array}$} \\
\hline \multicolumn{6}{|l|}{$\begin{array}{l}\text { 10. Si favorece a mi salud, consumiría } \\
\text { marihuana como tratamiento médico. }\end{array}$} \\
\hline \multicolumn{6}{|l|}{$\begin{array}{l}\text { 11. Siento curiosidad por consumir } \\
\text { marihuana de forma recreativa. }\end{array}$} \\
\hline \multicolumn{6}{|l|}{$\begin{array}{l}\text { 12. Consumiría marihuana con fines } \\
\text { medicinales si padeciera de dolores fuertes. }\end{array}$} \\
\hline \multicolumn{6}{|l|}{$\begin{array}{l}\text { 13. Si se diera la oportunidad consumaría } \\
\text { marihuana por diversión. }\end{array}$} \\
\hline \multicolumn{6}{|l|}{$\begin{array}{l}\text { 14. Si el doctor lo recomienda, consumiría } \\
\text { marihuana como medicamento. }\end{array}$} \\
\hline \multicolumn{6}{|l|}{$\begin{array}{l}\text { 15. Consumir marihuana de forma } \\
\text { recreativa puede ser agradable. }\end{array}$} \\
\hline \multicolumn{6}{|l|}{$\begin{array}{l}\text { 16. Creo que no tendría problema en } \\
\text { consumir marihuana con fines medicinales. }\end{array}$} \\
\hline & 1 & 2 & 3 & 4 & 5 \\
\hline
\end{tabular}

Obtención de puntajes:

Consumo y legalización medicinal: $(1+3+5+7+10+12+14+16) / 8$

Consumo recreativo: $(9+11+13+15) / 4$

Legalización recreativa: $(2+4+6+8) / 4$ 